\title{
Billing System Design Based on Internet Environment
}

\author{
Muzhir Shaban Al-Ani \\ Collage of Computer Science \\ Anbar Uiversity \\ Anbar, Iraq
}

\author{
Rabah Noory \\ Collage of Computer Science \\ Anbar Uiversity \\ Anbar, Iraq
}

\author{
Dua'a Yaseen Al-Ani \\ Collage of Computer Science \\ Anbar Uiversity \\ Anbar, Iraq
}

\begin{abstract}
This paper deals with the design of Internet billing system, in which it is possible pay invoices electronically. This approach is implemented via virtual banks, in which the process of money transfer can be implemented. In other hand many applications can be realize such as; deposit e-money, withdrawal e-money and determine account balance. A Gate way translator is used to apply authentication rules, security and privacy.
\end{abstract}

Keywords- Billing System, Internet Billing system , E-Commerce, E-bank, bill payment, Authentication, Security.

\section{INTRODUCTION}

Paper bills are now the primary channel of communication between companies and their customers. However, their potential for personalization is limited, and they are not interactive. If a customer wants to react to something in his paper bill - for example, to make a customer service inquiry or to order a new service - he must make a telephone call. Internet Billing promises far more than a new and inexpensive way to deliver billing information. Industry experts predict that Internet Billing will fundamentally change the way companies interact with their customers. Eventually, the Internet Bill will be an interactive entry to a host of additional services including customer self-care, automated sales one-toone marketing. The Internet Bill will become the gateway through which customers and companies have electronic one to one dialogs[1].

Businesses and consumers are banking on the Internet in more than one sense. Despite the early proliferation of electronic banking applications on private networks through dial-up services, most electronic banking applications have migrated to the Internet. Consumers will not be tied to one particular bank and its software, nor to a single terminal where the bank's own software must be installed. Banking on the Internet provides the flexibility of banking from any Internet access terminal using the now ubiquitous Web browser. Banking on the Internet can reduce the number of staff banks must maintain without having to make the investment in establishing private networks. The World Wide Web, or the Web, and its user-friendly, graphically rich browsers have made the Internet both friendly and accessible to the common desktop user at home and in the office [2].

The advancement of electronic banking or commonly known as e-banking, began with the use of ATMs and has included telephone banking, Direct bill payment, electronic fund transfer, online banking and other electronic transactions[3].

Banking services offered to consumers over the Internet will allow consumers to generate bank statements, check balances, transfer money between accounts, and authorize fund transfers to deposit money, to pay monthly bills, and to write personal checks. The Internet will provide a very competitive medium for banks to woo consumers. Consumers will be able to quickly and easily scan savings and loan rates and banking fees without having to interact with bank personnel.

Beyond home banking, consumers will be able to write electronic checks to online merchants that draw value directly from the consumer's own bank account rather than use a line of credit. The Internet will make banking a much more competitive environment in another critical aspect. Local banks will now be competing with national and international banks whose Internet presence removes barriers of physical distance. In addition, a number of "virtual" banks have now entered the market to compete with traditional banks for clients. The environment created by

Internet banking will present the vast array of services currently offered by banks in a form that is very convenient to consumers Commerce [2].

\section{E-COMMERCE}

Deep penetration and spread of Internet, lead to more electronic applications are becoming available. Electronic commerce (E-commerce) is one such enabling technology, which has wide spread utility touching almost everybody in society. It helps buyers and sellers, individuals and business, retail and bulk suppliers. In fact, e-commerce has very attractive features like anywhere, anytime shopping / banking ( 24 hours x 365 days) and no holidays, zero inventory, no middlemen, and so forth.

It helps customers to compare various products in the range and class, study their features/performance and make an informed decision about the emergence of e-commerce has created new financial needs that in many cases cannot be effectively fulfilled by the traditional payment systems. Recognizing this, virtually all interested parties are exploring various types of electronic payment (E-payment) system and issues surrounding e- payment system and digital currency [5]. 
The earliest example of e-commerce is Electronic Funds Transfer (EFT). This allows financial institutions to transfer funds between one another in a secure and efficient manner. Later, Electronic Data Interchange (EDI) was introduced to facilitate inter business transactions. However, early EDI systems were typically operated over special networks that are complex to set up and costly to administer. For these reasons, EDI has not been as widely deployed as expected. With the advent of Internet technologies and advanced cryptographic techniques, it is now feasible to implement e-commerce over a public network - the Internet. The development of the World Wide Web (WWW) greatly accelerates the development of ecommerce and expands its scope to cover different types of applications [6].

E-commerce includes activities such as establishing a Web page to support investor relations. In brief, e-commerce involves the use of information technology to enhance communications and transactions with all of an organization's stakeholders. Such stakeholders include customers, suppliers, government regulators, financial institutions, mangers, employees, and the public at large. E-commerce is a revolution in business practices.

If organizations are going to take advantage of new Internet technologies, then they must take a strategic perspective. That is, care must be taken to make a close link between corporate strategy and e-commerce strategy. Ecommerce, in a broad sense, is the use of computer networks to improve organizational performance. Increasing profitability, gaining market share, improving customer service, and delivering products faster are some of the organizational performance gains possible with e-commerce. E-commerce is more than ordering goods from an on-line catalog. It involves all aspects of an organization's electronic interactions with its stakeholders, the people who determine the future of the organization [7].

There are different types of e-commerce from perspective of the buyer and seller relationship, according to this relationship, e-commerce applications can be divided into the following four categories:

\section{A. Business-to-Consumer ( $B 2 C$ )}

In this case, the seller is business organization, whereas the buyer is consumer. This emulates the situation of physical retailing and so it is commonly called electronic retailing or consumer-oriented e-commerce. It frequently involves a temporary relationship and has relatively low volume of transactions and small payments.

\section{B. Business-to-Business ( B2B )}

In this case, the vendor and the buyer of the goods or services involved in a transaction are both organizations rather than individual customers.

In contrast to $\mathrm{B} 2 \mathrm{C}$ e-commerce, $\mathrm{B} 2 \mathrm{~B}$ is characterized by a number of features and these include high volumes of goods trade, prior agreements or contracts between the partners involved requiring a much higher level of authorization, taxation, and documentation and information exchange.

\section{Consumer-to-Consumer ( $C 2 C$ )}

This refers to situations where both the seller and the buyer are consumers. On line auctions provide an effective means for supporting $\mathrm{C} 2 \mathrm{C}$ e-commerce.

\section{Consumer-to-Business $(C 2 B)$}

This has perhaps been the area in which there has been the biggest growth in e-commerce. In this type of applications, a customer specifies his requirements in relation to the product or services he wants to the business represented by an ecommerce site which does a search over the Internet to explore the web sites that match these requirements and return the result to the customer [8].

\section{BILLING SYSTEM}

Billing systems are key competitive weapons for telecommunications companies [9]. A billing system is a combination of software and hardware that receives call detail and service usage information, grouping this information for specific accounts or customers, produces invoices, creating reports for management, and recording (posting) payments made to customer accounts. Billing systems are composed of interfaces (Network, Marketing, Customer Care, Finance, etc.), computers, software programs and databases of information. Computers are the hardware (computer servers) and operating systems are used to run the programs and process. Network interfaces are the hardware devices that gather accounting information (usage) from multiple networks, convert it into detailed billing records, and pass it on to the billing system.

Billing system use databases to hold customer information; usage call detail records, rate tables, and billing records that is ready to be invoiced. The key functional parts of a billing system include creating usage records, event processing, bill calculation, customer care, payment processing, bill rendering and management reporting. In addition to the basic billing system functions, billing systems share information with many other business functions such as sales, marketing, customer care, finance and operations.

Billing charges are determined by events that occur in a communication system. Billing events can originate from many sources: a media gateway, a media server, a content aggregator or a visited partner's network and they must be converted into a standard format. A typical billing process involves collecting usage information from network equipment (such as media servers, access devices and set top boxes), translating and formatting the usage information into records that a billing system can understand, transferring these records to the billing system, assigning charge fees to each event, creating invoices, receiving and recording payments from the customers[10].

Telecommunication companies need an effective and accurate billing system to be able to assure their revenue. Billing systems process the usage of network equipment that is used during the service usage into a single Call Detail Record (CDR). The billing process involves receiving billing records from various networks, determining the billing rates associated with the billing records, calculating the cost for each billing 
record, aggregating these records periodically to generate invoices, sending invoices to the customer, and collecting payments received from the customer. Billing system is very complex starting from network elements that generate usage to the billing system to usage collection, mediation, rating, and invoicing [11].

\section{ON-LINE BILLING SYSTEM}

Electronic billing is one of the fastest growing technologies for corporate law departments. Recent surveys indicate that roughly 15 percent of corporate legal departments require electronic bills from their law firms, and another 15 percent are considering it. If the person is a law firm with corporate clients, the person have probably seen acceleration in the number of requests from clients who want their bills submitted electronically. Choosing electronic billing and matter management systems are among the most important technology decisions that a law department can make, with significant potential consequences both positive and negative [12].

The concept of electronic billing is not new. Since the advent of the Internet, a small number of consumers have been using this electronic medium to pay bills online after receiving standard paper invoices via regular Postal Service. What is new in the electronic billing arena is the concept of electronic bill presentment. With electronic bill presentment, companies that send bills (billers) post consumers' statements to the Internet, enabling consumers to view the statements and make e-payments [1].

With ever increasing spread of Internet, Bill presentment and payment is becoming a new type of service area for periodic billers like Telephone Companies, Electricity etc. Internet based bill presentment and payment system converts billing centers from cost centers to revenue centers and for customers (payer) the system is a personalized service. Internet based bill presentment and payment system provides direct personalized communication channel between Billers and Payers, opens a new revenue channel by cross-selling advertisements. Drastic reduction of costs that are associated with paper based billing system. For customers or payers, receiving bills to payment of bills at one window through a Personal Computer, figure1 show on-line billing system.

Figure 2 Depicts overall workflow of the system step by step:

1. Customer gets an Electronic Cheque Book (e-Cheque Book) from his/her bank.

2. Customer sends registration request for online billing through biller's World Wide Web site.

3. Biller verifies credentials of the application and grants a subscription for online billing and sends user-id and password through e-mail or immediately when credentials are submitted. This enables the customer to view and pay bills.

4. Customer logs in to his/her online billing account of the biller's web site, verifies the bill details and pays with an electronic cheque (generated from the e-Cheque Book). The electronic cheque or e-cheque is sent to the Biller.

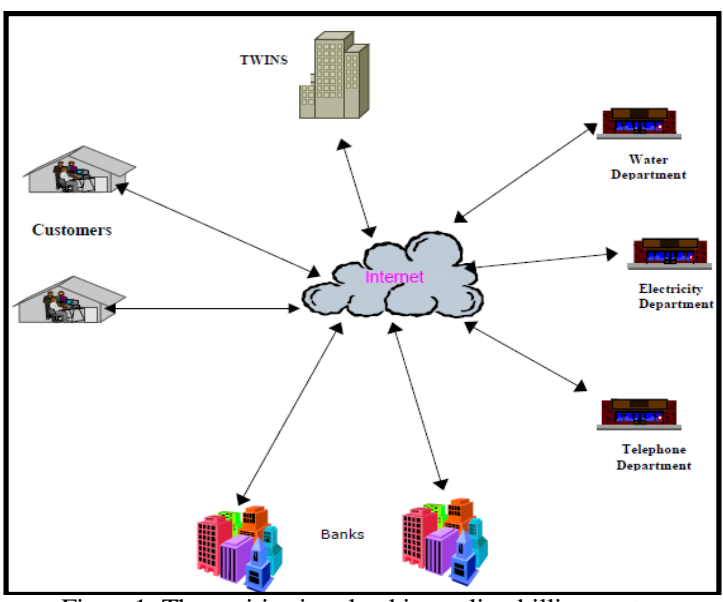

Figure1. The entities involved in on-line billing system

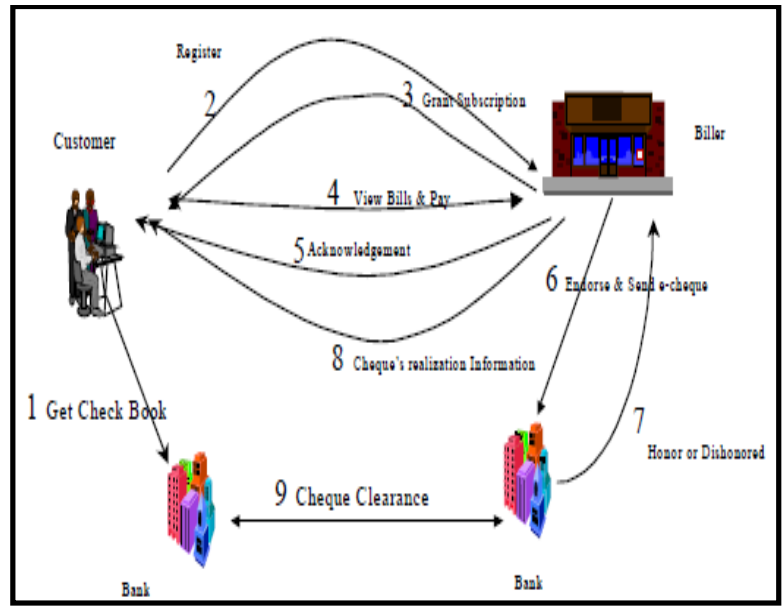

Figure 2: Internet billing system workflow

5. Biller system receives e-cheque and sends an acknowledgement for the received e-cheque.

6. Biller checks for validity of electronic cheques (received from customers), endorses, and sends them to bank for processing.

7. Bank (Biller's) validates the received e-cheques and sends them to the Customer's bank for clearance. Honored or dishonored information is sent to the Biller.

8. Biller's billing system updates the customer billing data based on cheque clearance status (as received from the bank) and sends appropriate information to the customer through electronic mail. The steps 1-3 above are done for registration, which is a one-time activity for a given customer, whereas steps 4-8 are used for viewing/paying bills, which is an ongoing activity [4].

\section{LITERATURE SURVEY}

There are many previous studies in the field of Internet billing system, below are some of these studies and their result are referred to:

- J Crookes (1996), the term adopted for the system is multiservice billing system (MSBS). The strategic business issues which have shaped the design of 
MSBS. It describes the scale and complexity of the problem which makes the construction of a multiservice platform such a difficult feat of software engineering. The concept of a common product model, which underpins the system's design, is introduced [9].

- NN Murthy, et al.(2000), In their paper, the authors presented a brief description of the technologies for e-commerce The authors also present TWINS (Twin Cities Information Network Service) test-bed application being developed as part of this project. TWINS, operational at twin cities of HyderabadSecunderabad, facilitates payment of various utility bill payment (like water, electricity, etc.) through a single window system. Payment of water bills through Internet using E-Cheque (Electronic Cheque) will be operational soon. This enables customers to pay their bills from anywhere, anytime. Thus, realizing the benefits of e-commerce to the citizens [4].

- Yang Bo, Liu Dongsu and Wang Yumin (2001), In their paper, the authors improved the e-payment system with a smart card proposed by S.Brands, and present an anonymity-revoking e-payment system. On the one hand, the customer's privacy cannot be compromised by the bank or by the payee. On the other hand, anonymity can be removed by a TTP with the help of the bank. In this case, the third party can link a payment to a corresponding withdrawal and prevent money laundering and blackmailing [13].

- EWB Team (2000), This document provided information regarding the use of the Extra Work Billing System (EWB). The document is organized with step-by-step instructions for each task to be accomplished using the EWB system. The EWB System may be accessed through the Internet using either Netscape Navigator or Internet Explorer [14].

- P.S. Barreto, et al. (2005). In their paper, the authors presented a discussion concerning the performance of four network scenarios for billing purposes. Using the results of packet losses in an experimental platform simulating a NGN (Next Generation Network) environment, the authors evaluate on each scenario the impact in the billing process with different traffic flows comparing the total revenue calculus for two billing schemes: (1) charging per packet and (2) reducing the value corresponding to undelivered packets. Our results show that the environments that use Differentiated Services are both convenient for costumers and service providers [15].

- Shiqun Li , et al. (2008). In their paper, the authors first identified some vulnerability in the mobile billing system. Then, the authors propose a fair and secure billing system based on a proper combination of digital signature and hash chain mechanism. The proposed system can achieve authentication, nonrepudiation, and fairness, which are desirable security requirements for an undeniable mobile billing system. [16].

- Albert Levi, Cetin Kaya Koc (2009), In their paper the authors proposed a new Internet e-payment protocol, namely CONSEPP (Convenient and Secure E-Payment Protocol), based on the account authority model of ANSI (American National Standards Institute) X9.59 standard. CONSEPP is the specialized version of X9.59 for Internet transactions (X9.59 is multi-purpose). In CONSEPP the authors propose a lightweight method to avoid the need for merchant certificates. Moreover, the authors propose a simple method for secure shopping experience between merchant and consumer. Merchant authentication is embedded in the payment cycle. CONSEPP aims to use current financial transaction networks, like Visa Net, Bank Net and $\mathrm{ACH}$ (Automated Clearing House) networks, for communications among financial institutions. No certificates (in the classical sense) or certificate authorities exist in CONSEPP [17].

- Giannakos Antoniou, et al. (2009), In their paper, the authors proposed an online payment scheme which uses the traditional e-payment infrastructure but which reveals no payment information to the seller. This is done with only an incremental increase in computational power [18].

\section{IMPLEMENTED BILLING SYSTEM}

The implemented system is intended to support all the banking operations (Direct bill payment; determine account balance, money transferred, withdrawal and deposit). Figure 3 shows the architecture of the implementation system.

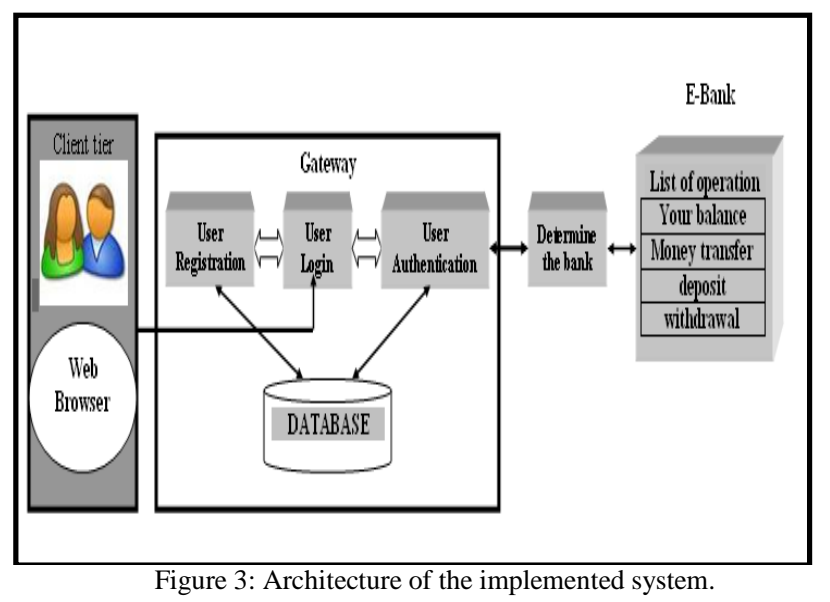

The implementation system consists of the following components:

\section{A. Client}

Clients (i.e. users and customers) can access to the implemented system from web browser (Internet explorer, firefox, etc) that provides a user interface that customer interacts this interface. The user must enters the name and the password to introduce to e-bank group . 


\section{B. Gateway}

Gateway is used to control several operations including user access to the system, check the validity and reliability of the user from use of the system and make sure that the user is one of the participants in the e-bank group. In addition make the registration process in the e- bank group for new customer. After all this, the user can log into the e- bank group. The gateway must authenticate the user before allowing him to enter any bank want to deal with it. User's authentication required the correct user name and password that must be entered at the login step. They will be checked against the stored ones in designated database, upon match, the user will grant the access to its account, hence, the provided services. Passwords will be stored at the designated database as plain text; an unauthorized access from local or remote user to the database can have an catastrophic damage.

She/he can use any username along with correct password to access, transfer, etc.... Therefore, Hash function MD5 (Message-Digest Algorithm) is used to generate message digest for all the passwords that will be stored in the database. At the authentication, the Hashed password will be checked rather than the plain text.

In the case that the user is a new customer, the information must enter which are full name, phone, E-mail, address, username, password and limitation. The limitation is that the amount of money determined by the user in the registration process, so the user cannot exceed this amount during withdraws or transfer funds. This mechanism used to add more protection for the process of withdrawal and transfer of funds. In case of exceeding the limitation specified, the proposed system making stop for the withdrawal operation and make sure the reliability and validity of user.

This information must entered by the user to be registered in the gateway and the bank chosen by the user. The Card number is resulting from taking the hash function MD5 and CRC32 function (Cyclic Redundancy Check) for some of information which is (ZIP for the country (This system applied three example of countries, Iraq, Paris, America), Full Name for the user, and code number for the bank). The implemented system produced the account number. The first customer is given the account number equal to one; the second customer is given the account number equal to two, etc. Figure 4 illustrates the user's registration.

\section{1) C. E-bank}

The user can choose any bank willing to deal with it. There are four operations for in the bank that is (account balance, withdrawal, deposit and money transfer) that the user want to make them and our DB is sensitive to the changes.

\section{2) Account Balance Operation}

The user needs to determine the his/her account balance, therefore he must enter the card number .when the server is matching between the user's card number and the card number in our designated database, the account balance is produced, and these operations as listed below:

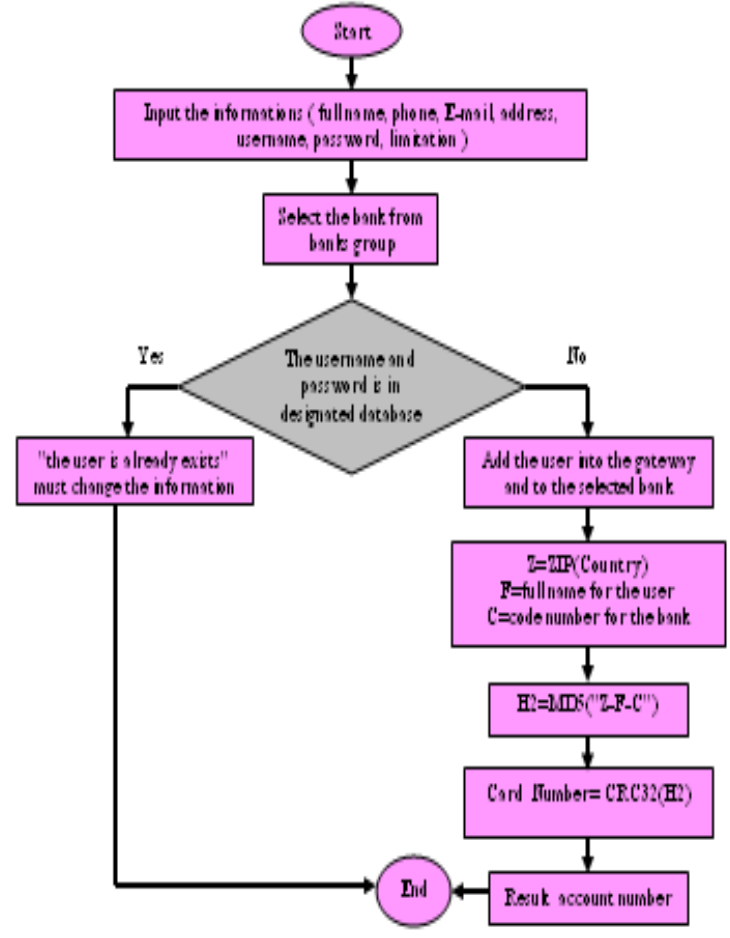

Figure 4: Flowchart of user's registration

\section{3) Money Transfer}

If the user wants to transfer money from any bank to another, then he must select the operation which is money transfer. The user can transfer the money from his/her account balance to another consumer by enters the several inputs (card number for the sender, account number for the Recipient, the amount of money to be transferred and determine the bank that receives the money).

The user can pay the bills for water, electricity, Telephone, etc., through the use of this proposed system and benefit from the money transfer service. Through a financial transfers between the user's bank and the banks that deal with Telephone Companies, water, Electricity, etc.

In the process of transferring funds from one bank to another, the mechanism is needed to convert the currency; where the process of conversion from one currency to another is through the program to determine Currency Exchange. Figure 5 illustrates the Money transfer.

\section{4) Deposit operation}

The user can select this operation, when he wants deposit the money in his/her account balance. He enters the card number and the amount of money which is wanted to add to his/her account balance.

\section{5) withdrawal operation}

The user must enter the card number and the amount of money who wants to withdraw from his/her account balance when he selects the withdrawal operation. 


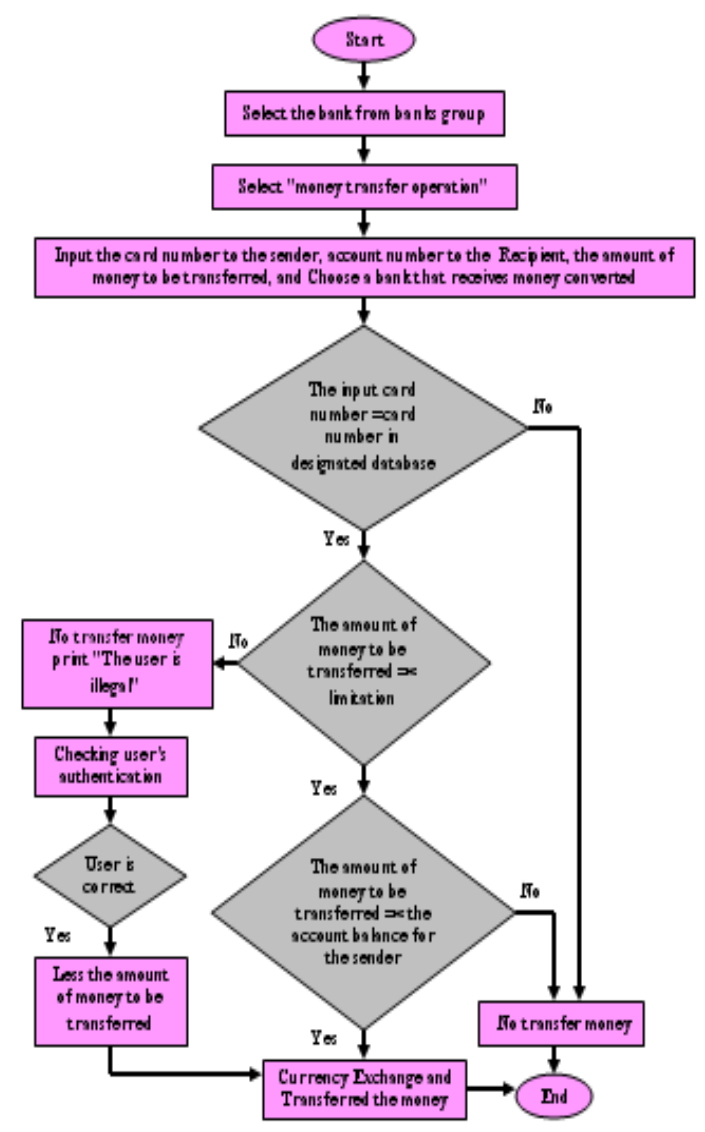

Figure 5: Overview of "money transfer" process

\section{RESULTS AND ANALYSIS}

The Internet billing system is implemented to satisfy the security requirements. The authentication process is done using hash function, CRC 32 function. The payment system work 24 hour a day, 7 days a week and any time anywhere.

The Implemented system leads to increase flexibility and efficiency of the payment process by reducing transaction process time and reducing cost.

The implemented system introduced many flexible interfaces such as main system interface (figure 6), user registration interface (figure 7) and E-bank services interface (figure 8).

\section{CONCLUSION}

In this paper, we implemented Internet billing system; by construction of virtual banks which perform the processes of banks. Some of the concepts of security have been applied in this system to protect the system from unauthorized access. The security issue is implemented via; encrypted passwords using hash function (MD5), the hash function (MD5) and CRC32. These functions are used to generate the card number; the amount of money transferred cannot exceed a certain imitation. Users can do their payment via E-bank any time anywhere, in which access time is reduced as possible.

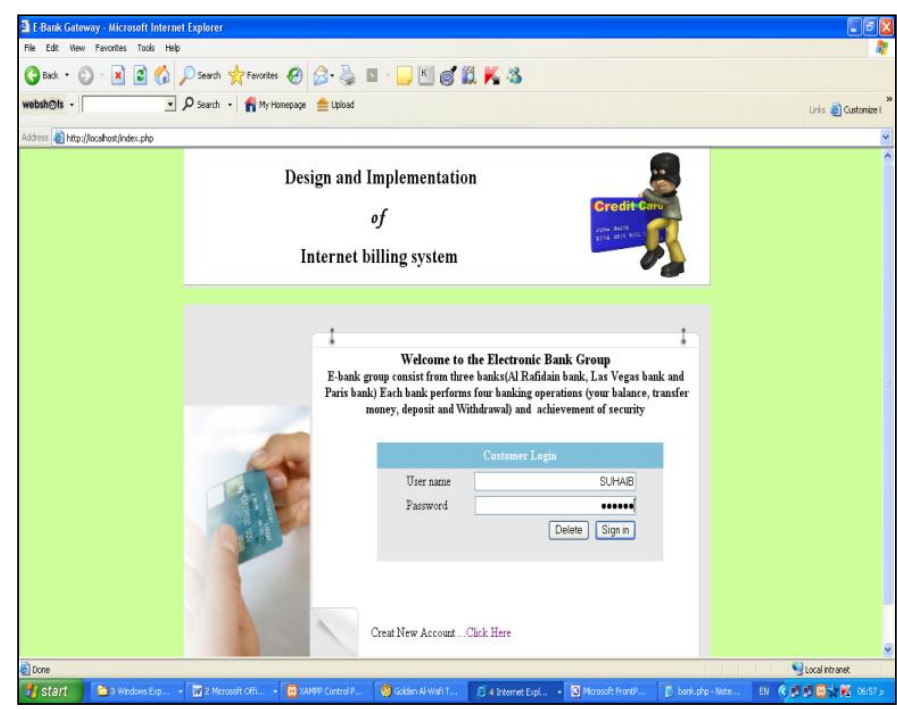

Figure 6: Main system interface

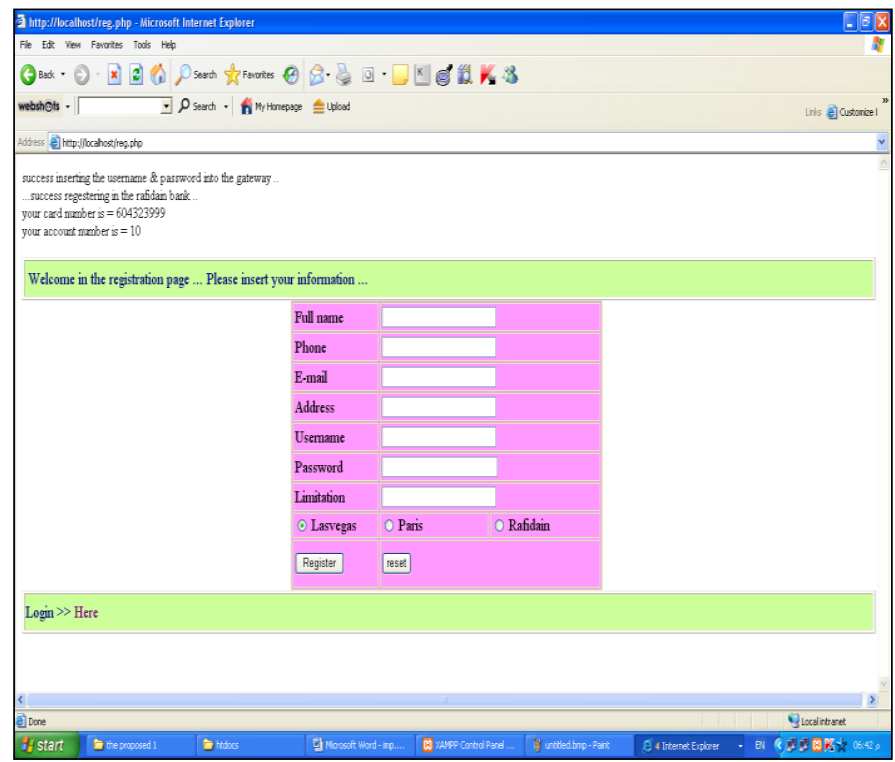

Figure 7: User registration interface

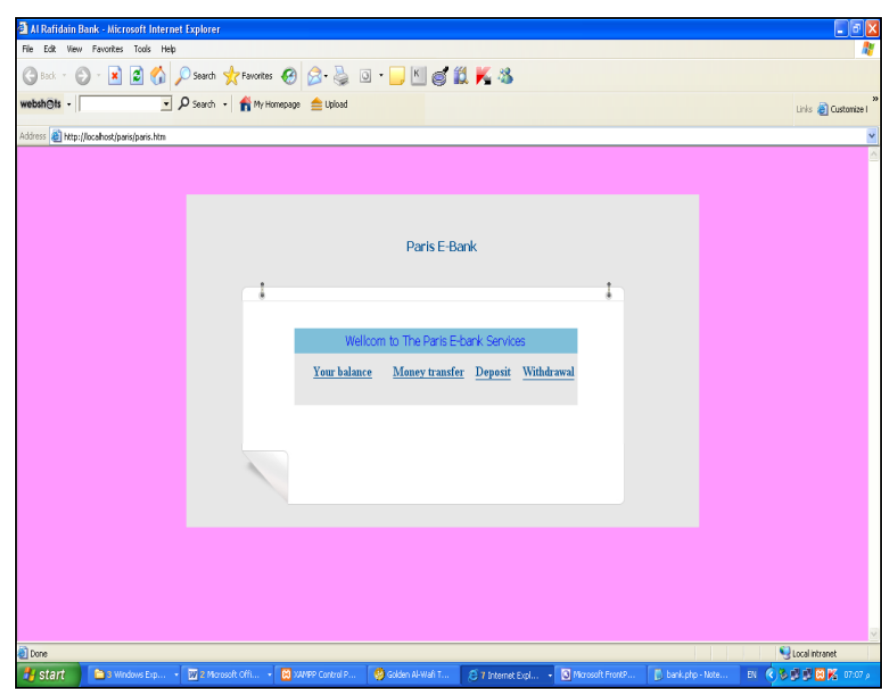

Figure 8: E-bank services interface 


\section{REFERENCES}

[1] Assimakopoulos Nikitas A., Anastasis N. Riggas \& Giorgos K. Kotsimpos, "A Systemic Approach for an Open Internet Billing System",2003,

http://www.afscet.asso.fr/resSystemica/Crete02/Assimakopoulos,\%20Ri ggas, \%20Kotsibos.pdf

[2] Ghosh Anup K., "E-commerce Security": Weak Links, Best Defenses, Wiley Computer Publishing, 1998.

[3] Singh Abhishek, OM Shankar, Vikash Kumar and Tapanray," Risk in E-Banking", CC BY-NC 3.0,2009, available at

http://www.scribd.com/doc/22356535/Risk-in-E-Banking-PDF,visited on July $16,2011$.

[4] NN Murthy, BM Mehtre, KPR Rao, GSR Ramam, PKB Harigopal, and KS Babu, "Technologies For E-Commerce: An Overview", CMC Center-R\&D, CMC Limited Old Mumbai Highway, Gachibowli Hyderabad - 500 019, Andhra Pradesh ,2000.

[5] Sumanjeet Singh," Emergence of Payment Systems In The Age Of Electronic Commerce:The State Of Art ", Global Journal of International Business Research Vol, 2, No, 2, 2009.

[6] Chan Henry, Raymond Lee, Tharam Dillon and Elizabeth Chang ,"Ecommerce Fundamental and Applications", Baffins Lane, Chichester, West Sussex, PO19 IUD, England,2001.

[7] Watson Richard T. , Pierre Berthon,Leyland F. Pitt and George M. Zinkhan, "ElectronicCommerce :The Strategic Perspective", Creative Commons Attribution 3.0 License, 2007.

[8] Media Abdul Razak Ali,M.Sc ,In a computer and software engineering department of the University of AL-mustansiriya, "Design and Implementation of SET Enabled E-commerce System",2005.

[9] Crookes J ,"Multiservice Billing System - a platForm for the future", BT Technol JVol 14 No 3 July 1996.
[10] Harte Lawrence, "Internet TV Billing Systems", Althos pupishing,2011, http://www.althos.com/tutorial/Internet-TV-station-tutorial-BillingSystems.html.

[11] Mostafa hatem, "Billing System : Introduction", codeproject, 2005, http://www.codeproject.com/KB/architecture/billing.aspx\#Introduction

[12] Thomas Rob, "Choosing an E-Billing System", published in I L T A December, 2005.

http://www,serengetilaw,com/news/serengetimeasuretwice,pdf.

[13] Bo Yang,Liu Dongsu and Wang Yumin, "An Anonymity-Revoking Epayment System with a Smart Card", springer-verlag, Volume 3, Number $4, \quad 4 \quad$ December 2001, http://www.springerlink.com/content/2uhetkje7a1pkljk/.

[14] EWB Team," Electronic Extra Work Billing System: Online Step -ByStep Instructions", Revision 2, ISSC, EWB Release 1.1 Instructions, January 12, 2001, http://www,dot,ca,gov/hq/esc/tollbridge/BenMar/006034/MaterialsHand out/EWB,pdf.

[15] Barreto P.S. , G. Amvame-Nze, C.V. Silva, J. S. S. Oliveira, H.P. de Carvalho, H. Abdalla Jr, A.M. Soares, and R. Puttini,"A Study of Billing Schemes in an Experimental Next Generation Network", SpringerVerlag Berlin Heidelberg 2005 , http://www.springerlink.com/content/r5nh3n0ebgf7w2h3/.

[16] Shiqun Li · GuilinWang · Jianying Zhou - Kefei Chen,"Fair and Secure Mobile Billing Systems", Springer Science+Business Media, LLC, 2008.

[17] Levi Albert and Çetin Kaya Koç "CONSEPP: Convenient and Secure Electronic Payment Protocol Based on X9.59", IEEE Computer Society Press, Los Alamitos, California, March 21, 2009, http://discuss.itacumens.com/index.php?topic=57564.0.

[18] Antoniou Giannakis, Lynn Batten, Shivaramakrishnan Narayan, and Udaya Parampalli, "A Privacy Preserving E-payment Scheme", Springer-Verlag Berlin Heidelberg 2009, http://www.springerlink.com/content/h2253738530vm061/ . 